\title{
CT-Guided Percutaneous Biopsy of a Mass Lesion in the Upper Presacral Space: A Sacral Transneuroforaminal Approach
}

\author{
Tilman Schubert $\cdot$ Augustinus L. Jacob • \\ Martin Takes · Thomas Menter • Andreas Gutzeit • \\ Sebastian Kos
}

Published online: 26 July 2011

(C) Springer Science+Business Media, LLC and the Cardiovascular and Interventional Radiological Society of Europe (CIRSE) 2011

To the Editors

The presacral region has unique anatomic and oncologic properties. It is occupied by loose connective tissue, nerves, blood, and lymphatic vessels [6]. Syed et al. [6] analyzed the histological diagnosis of 91 cases with sacral/ presacral mass lesions after biopsy. Lesions were predominantly neoplastic (70\%) with metastases and secondary tumors (77\%, plasma cell tumor, colorectal carcinoma, lymphoma, breast carcinoma, to name the most common) outbalancing primary neoplasms (23\%). The nonneoplastic lesions were preferentially inflammatory. The variety of mass lesions in the presacral space underlines that a histological analysis is essential to make the adequate therapy decision.

Minimally invasive biopsy of presacral mass lesions can be performed along different trajectories. The most common approaches are anterior or lateral transabdominal, transgluteal through the greater sciatic foramen, and anterolateral extraperitoneal [1,2]; however, alternative approaches, such as transosseous (transsacral or transiliac) [2, 3], precoccygeal [7], and endoscopically perineal [5], have been described. Nevertheless, lesions in the upper presacral space especially close to the os sacrum can be challenging to acquire histologic samples. In this context,

T. Schubert $(\bowtie) \cdot$ A. L. Jacob $\cdot$ M. Takes $\cdot$ S. Kos

Department of Radiology and Nuclear Medicine, University

Hospital Basel, Basel, Switzerland

e-mail: TSchubert@uhbs.ch

T. Menter

Department of Pathology, University Hospital Basel, Basel, Switzerland

A. Gutzeit

Department of Radiology, Cantonal Hospital Winterthur,

Winterthur, Switzerland we describe a sacral, transneuroforaminal approach. To our knowledge, this is the first report of obtaining a biopsy sample of a presacral lesion through this access. To minimize the risk of complications, we applied a computed tomography-based navigation system for procedure guidance.

\section{Case Report}

A 62-year-old woman was referred to our department with a 5-year history of malignant melanoma of the upper back. Annual positron-emission tomography/computed tomography (PET-CT) control using a combined PET-CT scanner (GE Medical Systems, General Electric Company, Fairfield, CT, US) revealed a fluor-deoxy-glucose (FDG)positive mass lesion in the upper presacral space. In light of the patient history, biopsy was immediately performed. Vulnerable structures, such as the sciatic nerve, the internal iliac artery, and the sigmoid colon along a caudocranial needle path, precluded a transgluteal approach through the greater sciatic foramen. Because the suspicious mass lesion was located immediately ventral to the right S1-neuroforamen, a transforaminal approach was chosen.

Percutaneous CT-guided intervention was performed in a 64-slice CT-scanner (Somatom Sensation 64, Siemens Medical Solutions, Erlangen, Germany) using a navigation system for percutaneous interventions (ActiViews, Haifa, Israel). The patient was positioned prone with arms fixed above his head. Conscious sedation (Midazolam/Dormicum and Alfentanil/Rapifen) was administered intravenously, and vital parameters were continuously monitored. For procedure planning, noncontrast $\mathrm{CT}$ of the pelvis was performed. By means of an X-ray-absorbing grid on the skin, the cutaneous entry point was chosen. A single-use 
reference pad with CT-visible fiducial markers was attached to the patient's skin. A navigation CT scan without contrast media was then performed and data were transferred to the navigation system, where the needle trajectory was planned. The cutis was locally anesthetized with $9 \mathrm{ml}$ of mepivacaine $1.5 \%$ diluted with $1 \mathrm{ml}$ of natrium bicarbonate $8.4 \%$. Along the prior planned trajectory and according to its lengths, a $17 \mathrm{G} 14.3 \mathrm{~cm}$ coaxial introducer needle (Medical Device Technologies Inc., Gainesville, FL) was chosen. A miniature, single-use video camera was then attached to the needle (Fig. 1). During navigation, the mapping of the camera and the probe are determined relative to the fiducial markers. The combination of the above mapping enables determination of the

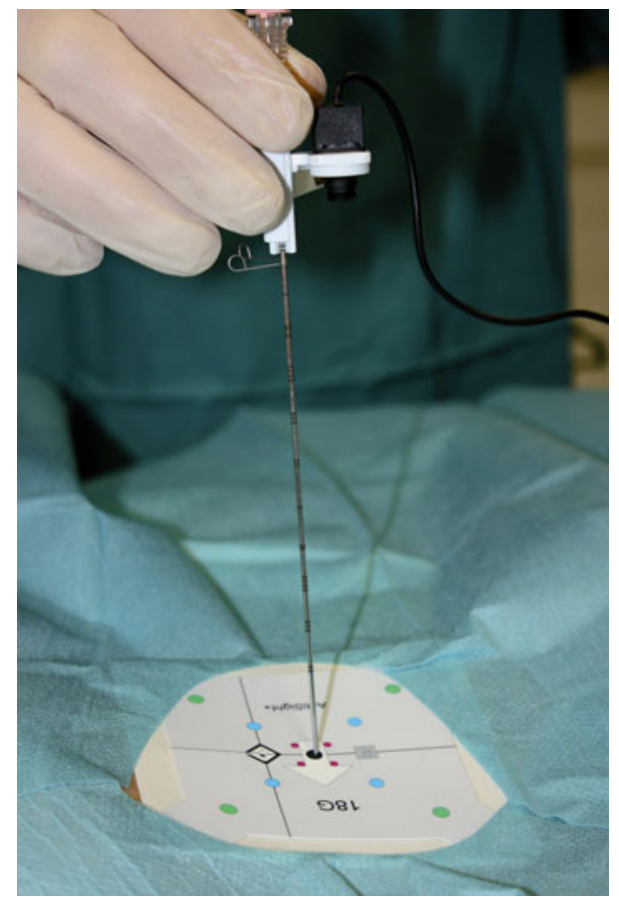

Fig. 1 Setup of the applied, CT-based navigation system. The fiducial markers on the reference pad on the patient's skin are captured by the miniature camera attached to the needle needle position relative to the actual target. The needle was then advanced in a single step to the dorsal ostium of the S1 neuroforamen (Fig. 2A). Before entering the S1 neuroforamen, the periosteum was locally anesthetized; however, only $2 \mathrm{ml}$ of bupivacaine $0.25 \%$ was administered to avoid nerve root block. After a low-dose CT control (slice thickness $3 \mathrm{~mm}$ ), the needle was slowly advanced through the neuroforamen without needing to correct the trajectory. During this procedure, the patient negated any radicular pain sensation. A further CT control showed correct placement of the needle tip at the target lesion. After removal of the inner stylet, an $18 \mathrm{G}$ 20-cm Menghini-type semiautomatic biopsy device (Biomol, H.S. Hospital Service, Rome, Italy) was inserted for biopsy. Five biopsy specimens were taken from different portions of the lesion by a slight movement of the coaxial needle tip in left-right and cranio-caudal directions (Fig. 2B, C). The solid specimens were deposited in a fixating solution and sent for histopathological analysis. The procedure lasted $53 \mathrm{~min}$ from the initial CT planning to the postinterventional control. The follow-up CT showed no signs of complications. The patient tolerated the procedure well and was monitored for an additional $6 \mathrm{~h}$ after the procedure without evidence of S1-palsy, paresthesia, or other complications. The combined morphologic and immunohistochemical diagnosis was that of a soft-tissue metastasis of the previously diagnosed malignant melanoma.

\section{Discussion}

The cytological and histological assessment of suspicious mass lesions is mandatory to allow potential tumor staging and therapy planning. Small lesions in the upper presacral space can be challenging for needle biopsy, because they are difficult to impossible to reach via a caudal or lateral approach. In those cases, and if in the effective direction, a transneuroforaminal approach is an alternative. The sacral transneuroforaminal approach is a common technique for electrical nerve stimulation for bladder dysfunction or
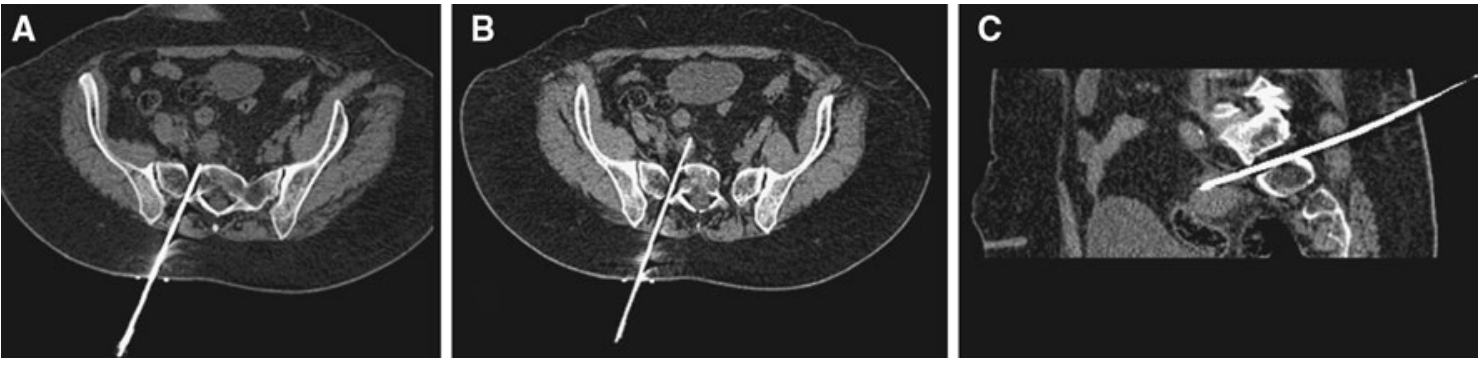

Fig. 2 A CT control after carefully advancing the needle through the S1- neuroforamen after application of a minimal amount of local anesthesia at the dorsal ostium to avoid nerve root block. To obtain the biopsy specimen, the needle tip was slightly moved in lateral and cranio-caudal directions $(\mathbf{B}, \mathbf{C})$ 
chronic pain management [4]; however, needle diameter is significantly less than that necessary to obtain biopsy specimen. The utilized 17-gauge $(1.4 \mathrm{~mm})$ coaxial needle is still small compared with the $\mathrm{S} 1$ neuroforamen. We measured $8.5 \mathrm{~mm}$ as the minimal diameter of the ventral ostium where the S1-nerve root leaves and $5.8 \mathrm{~mm}$ for the dorsal ostium, where only minor sensory skin branches leave. A navigation system for CT-guided biopsies ensures that the needle exactly follows the planned trajectory, especially in off-axial approaches. Therefore, the use of a navigation system minimizes the risk of injuring the nerve root due to intraforaminal correction of the needle path or reposition of the needle. To avoid nerve root injury while passing intraforaminally, we administered only a small amount of local anesthetic at the dorsal ostium of the neuroforamen to anesthetize the periosteum and still have patient feedback regarding pain or sensomotory sensations.

The costs of the applied system are \$75,000 USD, which includes training, service, and disposables for a year. Subsequent disposables amount to $\$ 300$ per procedure.

Acknowledgement This work was supported through an educational grant from Bracco Imaging S.p.A., Milano, Italy.

Conflict of interest The authors declare that there is no actual or potential conflict of interest in relation to this article.

\section{References}

1. Butch RJ, Wittenberg J, Mueller PR, Simeone JF, Meyer JE, Ferrucci JT Jr (1985) Presacral masses after abdominoperineal resection for colorectal carcinoma: the need for needle biopsy. AJR Am J Roentgenol 144:309-312

2. Gupta S, Nguyen HL, Morello FA Jr, Ahrar K, Wallace MJ, Madoff DC, Murthy R, Hicks ME (2004) Various approaches for CT-guided percutaneous biopsy of deep pelvic lesions: anatomic and technical considerations. Radiographics 24:175-189

3. Iguchi T, Asami S, Kubo S, Kin H, Katusi K, Sakurai J, Hiraki T, Kanazawa S (2007) CT-guided placement of a drainage catheter within a pelvic abscess using a transsacral approach. Cardiovasc Intervent Radiol 30:1277-1279

4. Jezernik S, Craggs M, Grill WM, Creasey G, Rijkhoff NJ (2002) Electrical stimulation for the treatment of bladder dysfunction: current status and future possibilities. Neurol Res 24:413-430

5. Nieuwenhuis DH, Gagner M, Consten EC (2009) The endoscopic perineal approach to the presacral space: an excision biopsy. J Laparoendosc Adv Surg Tech A 19:799-801

6. Syed R, Bishop JA, Ali SZ (2010) Sacral and presacral lesions: cytopathologic analysis and clinical correlates. Diagn Cytopathol doi: $10.1002 / \mathrm{dc} .21480$

7. Trambert JJ (1999) Percutaneous interventions in the presacral space: CT-guided precoccygeal approach-early experience. Radiology 213:901-904 